\title{
Review
}

\section{Progress in precision medicine in cystic fibrosis: a focus on CFTR modulator therapy}

The genetic multisystem condition cystic fibrosis (CF) has seen a paradigm shift in therapeutic approaches within the past decade. Since the first clinical descriptions in the 1930s, treatment advances had focused on the downstream consequences of a dysfunctional cystic fibrosis transmembrane conductance regulator (CFTR) chloride ion channel. The discovery of the gene that codes for CFTR and an understanding of the way in which different genetic mutations lead to disruption of normal CFTR function have led to the creation and subsequent licensing of drugs that target this process. This marks an important move towards precision medicine in $\mathrm{CF}$ and results from clinical trials and real-world clinical practice have been impressive. In this review we outline how CFTR modulator drugs restore function to the CFTR protein and the progress that is being made in this field. We also describe the real-world impact of CFTR modulators on both pulmonary and multisystem complications of CF and what this will mean for the future of CF care.

\section{Introduction}

Cystic fibrosis (CF) is a life-limiting multisystem condition first comprehensively described in the 1930s through autopsy studies observing mucus plugging in the pancreatic ducts of malnourished infants and the association of gastrointestinal disease with bronchiectasis [1]. This gave rise to recognition of a disease process characterised by thick secretions arising from mucous glands throughout multiple systems in the body with a high infant mortality rate due to malnutrition and respiratory failure. Subsequent discoveries lead to the recognition of $\mathrm{CF}$ as a genetic disease with an autosomal recessive inheritance pattern and high levels of excreted sweat chloride concentrations in affected persons, implicating an ion channel as the likely underlying defect. Identification of the CFTR gene, which codes for the cystic fibrosis transmembrane conductance regulator (CFTR) protein, occurred in 1989 [2-4], and to date, there are over 2000 recognised mutations in the gene although not all are considered to be definitively CF-causing [5].

Outcomes for individuals with CF have vastly improved over the decades, transforming the condition from one of childhood mortality to one of survival into the fifth decade and beyond. The cornerstone of these advances has been the development of multidisciplinary care focused in specialist centres with an emphasis on nutritional status and respiratory health (see figure 1). Early advances were particularly marked by the advent of supplemental pancreatic enzymes and nutritional support to treat exocrine pancreatic insufficiency.
Cite as: Tewkesbury DH, Robey RC, Barry PJ. Progress in precision medicine in cystic fibrosis: a focus on CFTR modulator therapy. Breathe 2021; 17: 210112

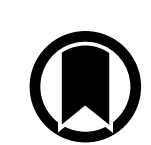

CrossMark 


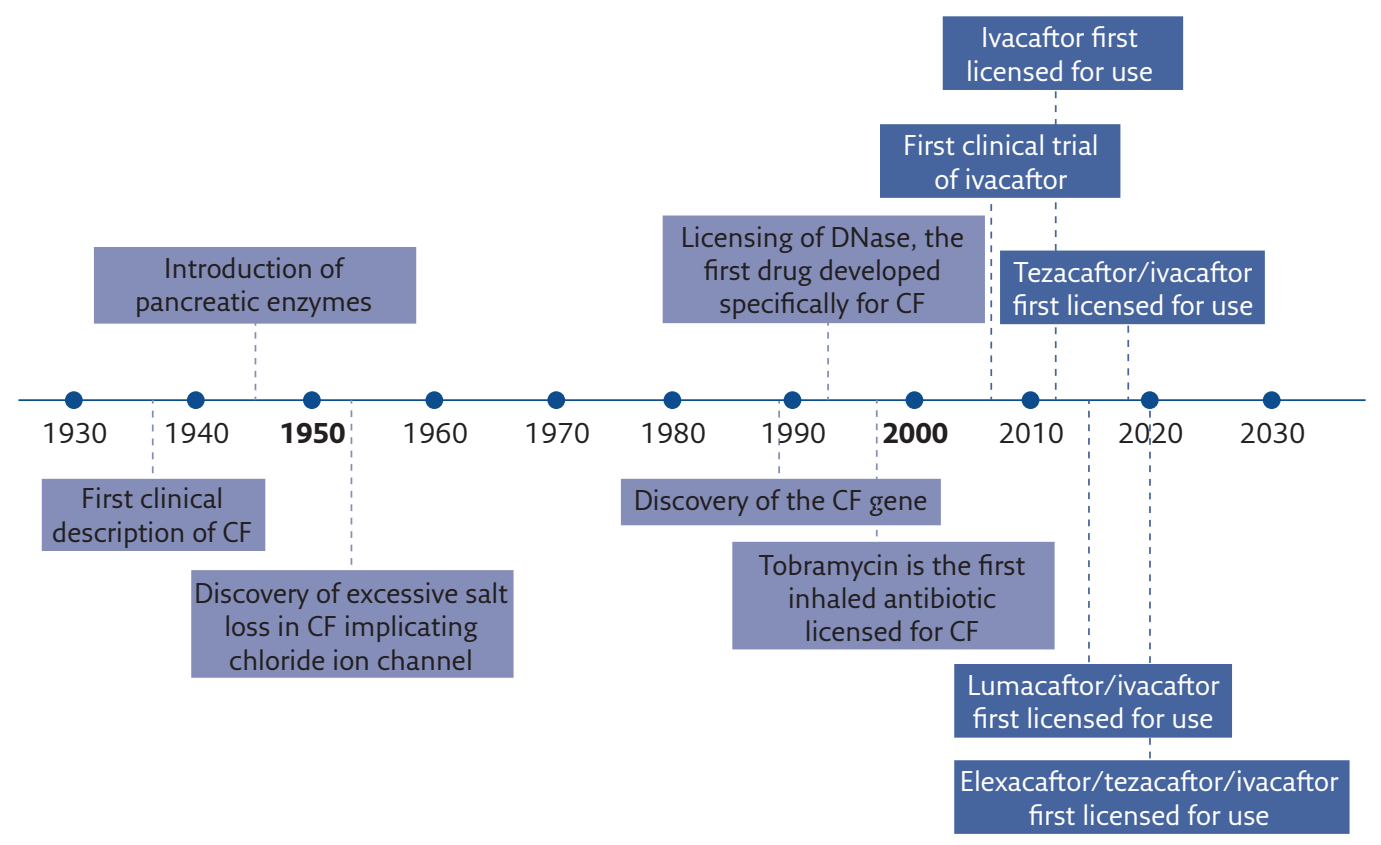

Figure 1 Timeline of treatment advances in CF.

Subsequently, as respiratory disease became more manifest, focused antibiotic therapy and airway clearance techniques were used to facilitate treatment of bronchiectasis and airways chronically infected with pathogens such as Pseudomonas aeruginosa.

The above treatments were similar in that they all targeted the consequences of end-organ damage resulting from the dysfunctional protein product of gene mutations. In the late 1980s the CFTR gene was identified, and further work elucidated the role of the CFTR protein as an ATP-binding cassette $(A B C)$ transporter-class ion channel. Research efforts focused on examining the individual consequences of each specific genetic mutation on the protein product, with four mutation classes initially described in 1993 [6]. Currently, there are broadly six classes of mutations which have been proposed [7]. Understanding the mechanism underlying protein dysfunction for each individual genetic mutation and indeed class provided targets for researchers to focus efforts on restoring function to the protein and thereby aiming to treat the root cause of CF, in turn carrying with it the hope that such an intervention would be accompanied by true disease modification. This review highlights the transformation of CF therapeutics into an era of precision medicine and will focus on progress to date and the clinical outcomes described in clinical practice.

\section{The CFTR gene and CFTR protein}

The CFTR gene is located on chromosome 7, consisting of 27 coding exons spanning over
$250 \mathrm{~kb}$. This gene codes to produce the CFTR protein which is expressed at the surface of epithelial cells. The CFTR protein is a member of the $A B C$ transporter proteins and consists of two membrane spanning domains, two cytosolic nucleotide-binding domains and a regulatory $(R)$ domain which contains phosphorylation sites. The CFTR protein functions predominantly as a chloride and bicarbonate channel, although it has roles in transporting other ions and modifying the function of other ion transporters. Ultimately, this positions CFTR as a crucial regulator of transepithelial fluid and electrolyte transport and dysfunction of the protein can lead to dehydrated liquid layers predisposing airways to chronic infection and bronchiectasis alongside other manifestations such as pancreatic insufficiency and liver disease.

There have been over 2000 mutations described in the CFTR gene although not all have been determined to disrupt protein function sufficiently to be considered "disease-causing". Other mutations have been determined to be of "varying clinical consequence" (VCC) due partially to altered penetrance and expressivity and may cause disease in certain individuals. Notwithstanding this, the most common CFTR mutations have been well described and furthermore the molecular consequences of these mutations have been better understood (figure 2).

Mutations can be broadly grouped into classes according to the way in which CFTR activity is disrupted. Class I includes nonsense and frameshift mutations leading to the creation of premature stop codons (PTCs) which result in no functional CFTR being created within cells. Class II mutations (the most common being Phe508del) result in abnormal CFTR protein 


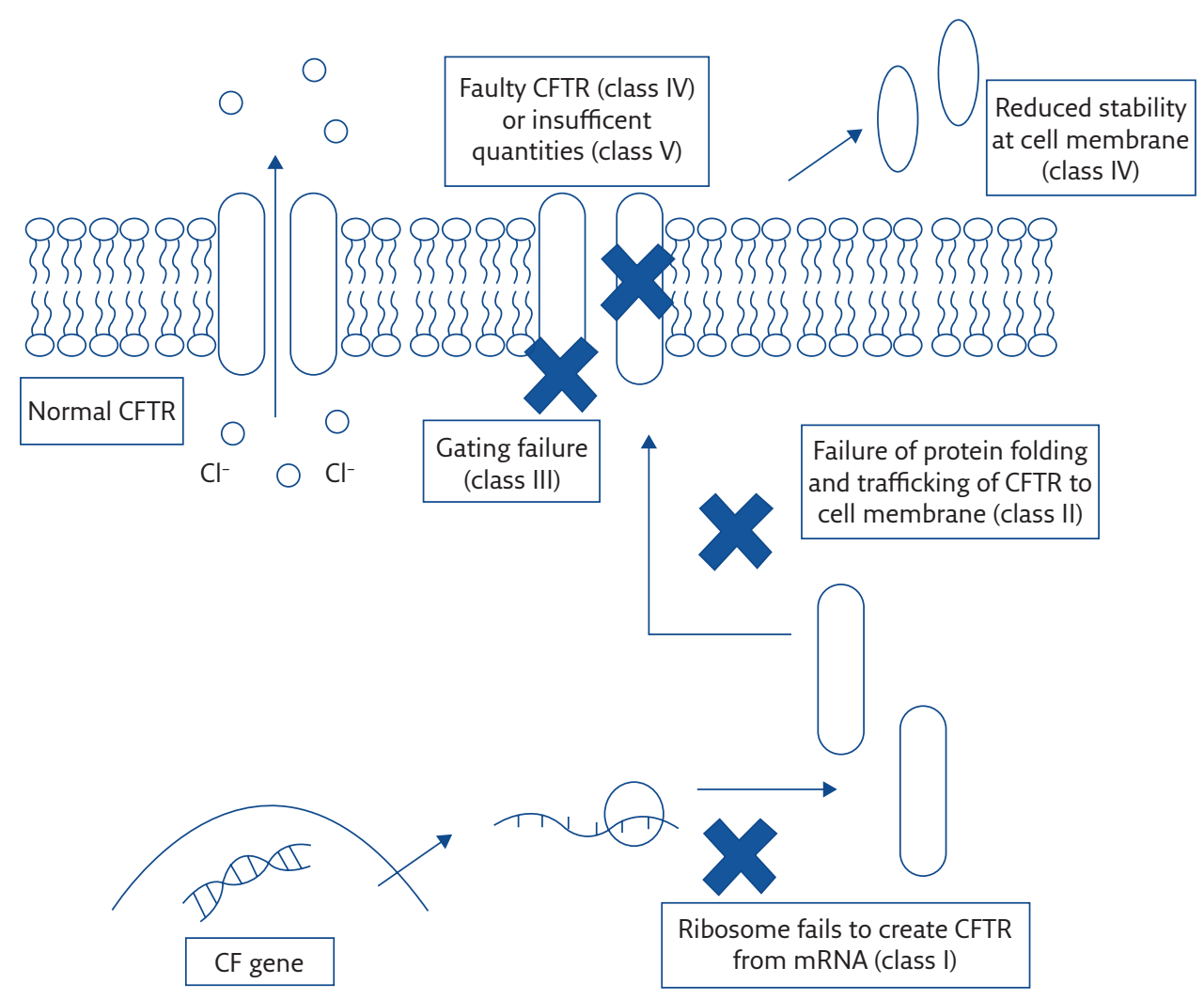

Figure 2 Mechanisms by which mutations in the CF gene can disrupt normal CFTR function.

processing leading to intracellular protein degradation. Class III mutations, often referred to as "gating" mutations, result in protein correctly transported to the cell surface but with a defective channel opening. Class IV-VI mutations result in CFTR being expressed at the cell surface but exhibiting reduced function because of altered conductance of the channel (class IV), reduced quantity at the cell surface (class V) or an unstable protein (class VI). Although useful to understand the consequences of individual mutations, this classification is not definitive with many mutations leading to defects of more than one class thereby requiring more than one defect to be targeted if efforts at restoring function are to be realised [8]. Certain generalisations can be made about mutations belonging to certain classes. Individuals homozygous for mutations denoted to be classes I-III will usually have a more severe phenotype with relatively little, if any, functioning CFTR protein. Individuals who carry at least one mutation in class IV-VI will often present with a less severe phenotype, often having pancreatic sufficiency and historically a later age at diagnosis. A further development of this has been the utilisation of the terms "minimal function" (MF) and "residual function" which incorporate the level of CFTR function. With regard to MF mutations, this terminology is also used to denote that these mutations are not responsive to current licensed modulator therapy.

\section{Approaches to precision medicine in CF}

Since the discovery of the CFTR gene in 1989 and with the development of better understanding of function of the CFTR protein, researchers have focused on restoring functional CFTR protein. Fundamentally this can occur in a number of ways: 1 ) by restoring a functional gene into epithelial cells, 2) by repairing the gene defect using gene editing strategies, 3 ) by using medications to enable ribosomes to "ignore" specific genetic mutations, 4) RNA-based therapies to either deliver CFTR-encoding mRNA or alternative approaches to either suppress mutant RNA or repair RNA, 5) the use of small molecules to enhance or restore function to aberrant protein, and 6 ) the use of medications to enhance or suppress the activity of alternative ion channels to negate the effect of CFTR dysfunction. While progress has been made in all fields the progress in the use of small molecules to restore function to aberrant protein has, up to this point, surpassed other approaches with a number of compounds now in everyday clinical use (table 1). Approximately $90 \%$ of individuals with CF worldwide will harbour at least one gene that produces aberrant protein which can be targeted by a new class of small molecules known as CFTR modulators. 
Table 1 CFTR modulators licenced for use by the European Medicines Agency (EMA)

\begin{tabular}{|c|c|c|c|}
\hline Modulator & Year of EMA licensing & Age group & Genetics \\
\hline \multirow[t]{4}{*}{ Ivacaftor } & 2012 & $\geq 6$ years & Heterozygous G551D \\
\hline & 2015 & $\begin{array}{l}\geq 2 \text { years } \\
\geq 18 \text { years }\end{array}$ & $\begin{array}{l}\text { As above } \\
\text { Heterozygous R117H }\end{array}$ \\
\hline & 2019 & $\geq 6$ months & As above \\
\hline & 2020 & $\geq 4$ months & $\begin{array}{l}\text { Heterozygous } \mathrm{R} 117 \mathrm{H} \\
\text { Heterozygous for gating mutations } \#\end{array}$ \\
\hline Lumacaftor/ivacaftor & 2015 & $\geq 12$ years & Homozygous Phe508del \\
\hline Tezacaftor/ivacaftor & 2020 & $\geq 6$ years & As above \\
\hline \multirow[t]{2}{*}{$\begin{array}{l}\text { Elexacaftor/ } \\
\text { tezacaftor/ivacaftor }\end{array}$} & 2020 & $\geq 12$ years & $\begin{array}{l}\text { Homozygous Phe } 508 \mathrm{del} \text { or Phe } 508 \mathrm{del}+\text { minimal function } \\
\text { mutation }\end{array}$ \\
\hline & 2021 & $\geq 12$ years & Heterozygous Phe508del \\
\hline
\end{tabular}

\section{CFTR modulators}

High-throughput screening was used to identify compounds that could improve protein function in in vitromodels. This has led to the development of small molecules known as CFTR modulators which are an orally bioavailable class of compounds targeting separate aspects of CFTR protein dysfunction [9]. These compounds can be grouped according to their function: potentiators, correctors, stabilisers, and amplifiers. A significant advantage of these therapies being orally bioavailable is that restoration of function occurs in a systemic manner and can address some of the multisystem consequences of CF.

\section{Potentiators}

CFTR potentiators act by increasing the probability that the CFTR chloride channel is in an "open" state, thereby increasing passage of chloride ions through the channel. This mechanism of action requires the presence of CFTR protein on the cell surface. The Gly551Asp mutation is the archetypal mutation in class III and CFTR potentiators were first tested in individuals heterozygous for this mutation and another CF-causing mutation.

The phase 2 study of ivacaftor (VX-770) in 39 subjects established safety of this compound and also provided encouraging data regarding efficacy with increases in lung function and quality of life [10]. Importantly, by evaluating the effect of these compounds on sweat chloride levels it could be established that these findings resulted from partial restoration of CFTR protein function, as sweat chloride is an important biomarker of CFTR protein function. Subsequent clinical trials showed a marked improvement in forced expiratory volume in $1 \mathrm{~s}\left(\mathrm{FEV}_{1}\right)$, body mass index (BMI) and quality of life, achieving outcomes previously unreported in CF clinical trials (see table 2) [11]. On the basis of these results, ivacaftor was licensed in the European Union and USA in 2012 for individuals with CF 12 years and older who carry at least one copy of the Gly551Asp mutation.

Studies with ivacaftor were conducted to explore its use in other mutations causing CF. Due to its noted effects in vitro on wild-type CFTR, it followed that its use could be expanded to improve function of CFTR protein which was limited due to other CF-causing mutations [11]. In vitro models suggested that a subset of individuals carrying the most common CFTR mutation Phe508del may also respond to ivacaftor, although theoretically it was believed to be unlikely to translate to clinical benefit as the major defect conferred by this mutation is of processing and trafficking with very little protein reaching the cell surface. FLume et al. [12] confirmed this suspicion in a phase $2 b$ study in 
Table 2 Clinical outcomes from major clinical trials in CF

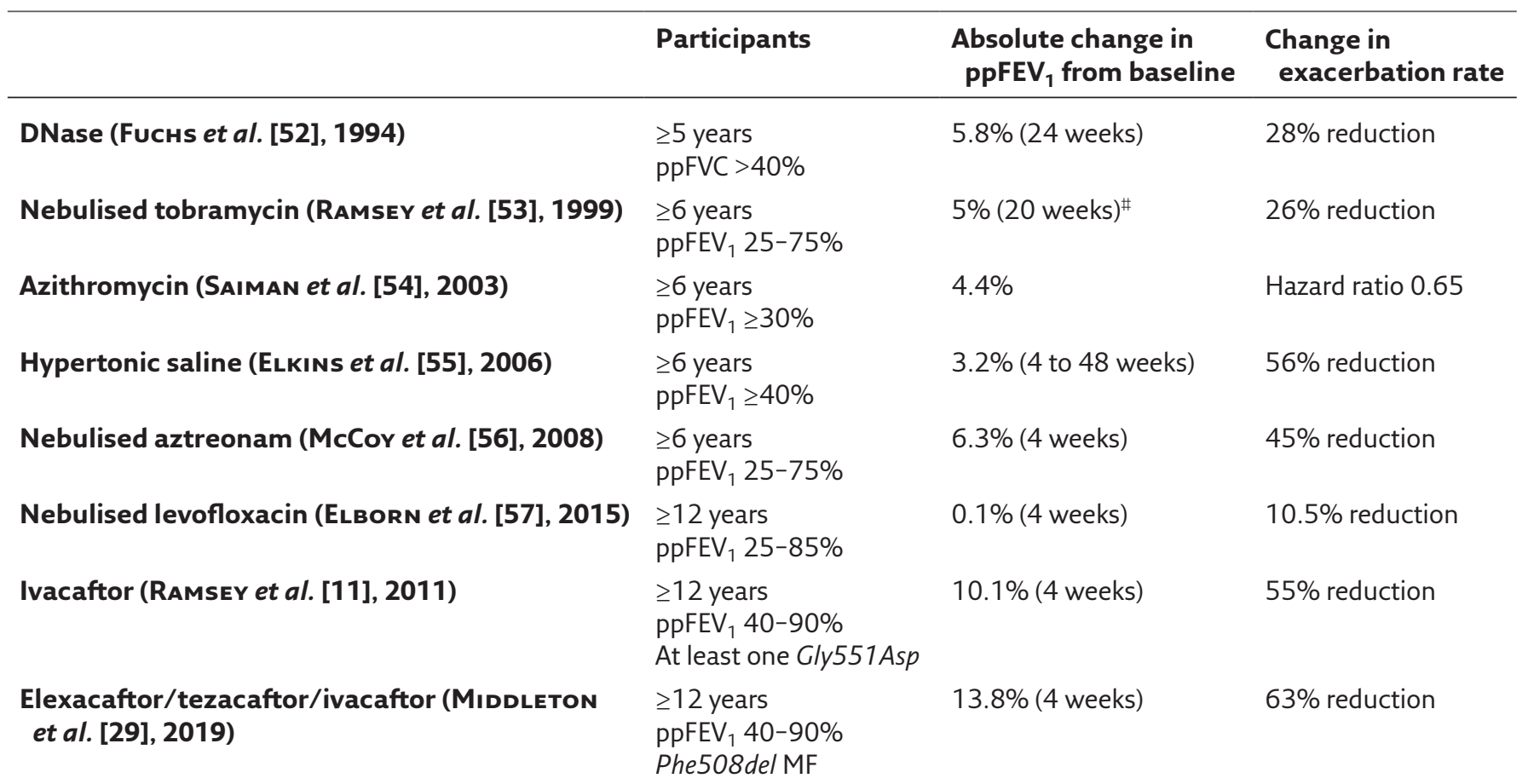

$\mathrm{ppFEV}_{1}$ : percentage predicted forced expiratory volume in $1 \mathrm{~s}$; ppFVC: percentage predicted forced vital capacity. \#: approximated using data from supplementary appendix on absolute change in $\mathrm{ppFEV}_{1}$ rather than change from baseline.

individuals homozygous for this mutation which demonstrated a minor change in sweat chloride, but no appreciable clinical benefit meaning that alternative strategies would be required to address this mutation and provide therapeutic options for the majority of people with CF (PwCF).

Clinical trials in PwCF carrying other mutations, however, did confirm the utility of ivacaftor in individuals who have protein on the cell surface. An 8-week trial of ivacaftor in PwCF 6 years and older carrying a class III ("gating") mutation other than Gly551Asp demonstrated substantial efficacy, which was similar to that of the original pivotal trials [13]. A clinical trial for individuals heterozygous for the mutation Arg117His provided mixed results with the overall data failing to demonstrate efficacy in terms of lung function, although in a prespecified subset analysis of adult subjects, ivacaftor therapy led to significant and clinically relevant improvements [14]. This apparent anomaly may have been due to the numbers in the trial and the relative outcomes for individuals may have placed an undue influence on outcome, but it should also be noted that this mutation is one associated with varying clinical consequences and may not be disease causing in all individuals.

Ivacaftor has been licensed according to the results of these trials with subsequent clinical trials in younger age groups now extending the licence to infants as young as 4 months old in Europe. It is notable that there is a discrepancy in licensing in different regions with a larger number of mutations licensed for ivacaftor in the USA, where the US Food and Drug Administration approved this compound based on results from in vitro studies [15].

A deuterated form of ivacaftor (deutivacaftor) demonstrates increased stability compared with ivacaftor [16] and would allow recipients to take a potentiator once daily. Results from phase 2 trials comparing efficacy and safety profiles have yet to be reported. Other potentiators, such as ABBV974, have also been shown to potentiate CFTR to a similar degree to ivacaftor but with no additional or synergistic effects, suggesting a common mechanism [17]. A further potentiator, ABV-2451 (previously GLPG-2451), has completed phase 1 trials but formal results are not currently published.

CFTR potentiators may even have use outside the realm of CF. The novel potentiator QBW251 (icenticaftor) demonstrated modest improvements in lung function for CF adults with class III or class IV mutations [18], but has also been trialled in COPD to target cigarette smoke-induced CFTR dysfunction. In phase 2 trials, COPD patients treated with icenticaftor demonstrated some improvements in $\mathrm{FEV}_{1}$ and sputum bacterial colonisation [19], highlighting acquired CFTR dysfunction as a novel therapeutic target in COPD.

\section{Correctors}

The need to expand the applicability of CFTR modulator drugs to more PwCF (most notably 
those carrying the Phe508del mutation) led to the development of a further class of CFTR modulators. CFTR correctors target abnormal protein folding and improve CFTR expression at the surface membrane. Several potential corrector compounds were identified by high-throughput screening that partially corrected for the abnormal protein folding and trafficking caused by Phe508del, although many were found to be unsuitable for clinical practice due to cytotoxicity [20]. Lumacaftor demonstrated an ability to restore CFTR function in Phe508del expressing cells [21] and was the first corrector to be licensed for use (in combination with ivacaftor) in 2015 after demonstrating a modest improvement in $\mathrm{FEV}_{1}$ as well as a significant reduction in pulmonary exacerbation rate for individuals homozygous for Phe508del in clinical trials [22]. Challenges with the use of lumacaftor existed, however, due to its potential for drug-drug interactions (notably with the oral contraceptive pill) and also some concerns regarding adverse respiratory symptoms, particularly in individuals with lower lung function.

The corrector tezacaftor was developed with the objective of improved pharmacokinetics and tolerance. In the pivotal phase 3 trial, when combined with ivacaftor, comparable clinical outcomes to lumacaftor/ivacaftor in Phe508del homozygotes were demonstrated [23]. This dual combination therapy was also further studied in individuals heterozygous for the Phe508del mutation and different classes of other mutations. In individuals heterozygous with a MF mutation the combination failed to result in clinical efficacy suggesting that this dual combination was insufficient to confer benefits for those carrying a single Phe508del allele and determining the need for further compounds to improve efficacy [24]. Similarly, in those heterozygous for a "gating" mutation, a phase 3 randomised controlled clinical trial did not report on improvements over and above those experienced with ivacaftor monotherapy [25]. In contrast, a phase 3 crossover trial looking at the effects of ivacaftor and tezacaftor/ivacaftor on individuals heterozygous for a residual function mutation did identify a benefit with ivacaftor monotherapy and a statistically significant improvement with the addition of tezacaftor [26]. Importantly, in individuals who had stopped lumacaftor/ivacaftor secondary to adverse events, tezacaftor/ivacaftor was well tolerated and associated with modest clinical benefit indicating a superior tolerability of this dual combination therapy [27].

More recently, tezacaftor/ivacaftor has been combined with a further corrector (elexacaftor) that improves CFTR protein folding and trafficking via different mechanisms. Clinical trials for elexacaftor/ tezacaftor/ivacaftor demonstrated a marked improvement in percentage $\mathrm{FEV}_{1}$ at 24 weeks as well as a significant reduction in sweat chloride concentrations, pulmonary exacerbation rate and improvement in quality-of-life scores [28-30]. As one trial included individuals heterozygous for a MF mutation these results meant the goal of developing highly efficacious therapy for the vast majority of individuals who carry at least one copy of the Phe508del mutation was realised. The substantial clinical improvements reported exceeded any seen in previous trials of CF medications (see table 2). A phase 3 trial has also demonstrated an additional benefit with this triple compound over and above that of either ivacaftor monotherapy or tezacaftor/ ivacaftor therapy for individuals who carry either a "gating" or residual function mutation on their second allele [31].

Several other correctors have demonstrated an ability to restore functional expression of CFTR and are undergoing evaluation in clinical trials. These include correctors developed by Abbvie/ Galapagos such as ABBV-2222 (galicaftor) which was shown to enhance CFTR activity in individuals homozygous for Phe508del or heterozygous with a gating mutation, although this did not translate to improvements in clinical end-points [32]. Trials are in progress to evaluate galicaftor in combination with other modulators, as well as trials evaluating other "third-generation" correctors such as PTI-801 (posenacaftor) and VX-121.

\section{Stabilisers}

In addition to disrupted trafficking of CFTR to the cell membrane, Phe508del also results in reduced stability at the cell membrane. So called "stabilisers" target class VI mutations as well as Phe508del by aiming to improve stability of CFTR at the membrane. Several methods have demonstrated an ability to improve CFTR stability in vitro including hepatocyte growth factor [33], activation of exchange factor directly activated by cAMP 1 [34], and inhibition of S-nitrosoglutathione reductase [35]. Cavosonstat is a stabiliser that works through inhibition of S-nitrosoglutathione reductase; however, in phase 2 clinical trials it did not demonstrate any improvement in lung function or sweat chloride concentration when combined with lumacaftor/ivacaftor (ClinicalTrials.gov identifier: NCT02589236) or ivacaftor (NCT02724527) and clinical development was halted.

\section{Amplifiers}

Finally, amplifiers aim to target CFTR dysfunction by increasing mRNA stability and therefore increasing the number of CFTR proteins synthesised and enhancing translation. Although they do not target specific mutation classes, they are only effective against mutations where either a functioning or modifiable CFTR protein is produced, meaning that they would have little effectiveness in class I mutations for example. Compounds such as nesolicaftor have demonstrated an ability to increase expression of CFTR [36] and trials are 
underway to evaluate clinical effectiveness, either when combined with other amplifier compounds (ClinicalTrials.gov identifier: NCT03500263) or modulators from different classes (such as tezacaftor/ivacaftor; NCT03591094).

\section{Outcomes with CFTR modulators}

\section{Acute pulmonary effects}

The initial trials of modulators focused primarily on their pulmonary effects, as progressive loss of lung function and respiratory exacerbations are the predominant source of morbidity and mortality in CF. Moreover, lung function tests and respiratory exacerbations provide useful, specific, measurable and quantifiable end-points for clinical trial outcomes and have been determined by licensing authorities as the key outcomes of interest. In the landmark trial in individuals heterozygous for Gly551Asp, ivacaftor monotherapy resulted in an improvement from baseline of around 10 percentage points in the $\mathrm{ppFEV}_{1}$ after 24 weeks of treatment [11]. This treatment effect was apparent just 15 days after initiating therapy and was sustained at 48 weeks. In addition, therapy led to a $55 \%$ reduction in pulmonary exacerbations over the 48-week study period, and a clinically significant improvement in scores on the respiratory symptoms domain of the Cystic Fibrosis Questionnaire-revised (CFQ-R; a widely used, standardised scale used to measure the effect of symptoms on patients' quality of life) [11].

The results with dual combination therapy were encouraging, particularly with relation to the reported reduction in pulmonary exacerbation events. However, the limited efficacy in comparison to what had been previously witnessed in the subset of individuals carrying mutations responsive to ivacaftor monotherapy underlined the need for further drug development. The results of trials of triple therapy have been considered to realise the hope that effective treatment could be achieved for the vast majority of PwCF.

Elexacaftor/tezacaftor/ivacaftor resulted in an improvement in $\mathrm{ppFEV}_{1}$ of 14 points at 24 weeks versus placebo in Phe508del heterozygotes [29], and 10-point improvement at 4 weeks versus tezacaftor/ivacaftor in Phe508del homozygotes [28]. Furthermore, elexacaftor/tezacaftor/ ivacaftor led to a $63 \%$ lower annualised rate of pulmonary exacerbations and a dramatic 20-point improvement in CFQ-R respiratory domain score versus placebo in Phe508del heterozygotes [29]. Increasing numbers of observational studies of these compounds in clinical practice are also encouraging. Substantial improvements in lung function have been witnessed in cohorts of patients with severe baseline lung disease who would have been considered too "unwell" to participate in clinical trials. Case series have observed a reduction in the need for noninvasive ventilation to manage respiratory failure and a reduction in the number of PwCF actively listed for lung transplantation [37].

\section{Longitudinal pulmonary outcomes}

With a change of focus to addressing the underlying cause of CF, it follows that the ambition with these therapies is to fundamentally alter the disease trajectory. With relation to pulmonary disease specifically the aim would be to alter the trajectory of lung function decline longitudinally in those with established disease and to prevent the development of new complications or even lung disease if the ability to treat early in life is realised. It is currently too early to establish the true longterm effectiveness of triple therapy; however, some evidence may be gleaned by studies of ivacaftor monotherapy in individuals with Gly551Asp, which was previously the benchmark in terms of CFTR modulation. In a comparison between trial participants and registry matched control subjects with ineligible genetic mutations, SAWICKI et al. [38] reported an attenuated rate of decline in lung function in patients on ivacaftor. Registry studies have similarly been informative with sustained improvements in ivacaftor treated patients over and above those not eligible for therapy. However, it is important to note that lung function decline has continued [39]. Data from the Irish registry suggests that differential effects may be seen depending on the age at which therapy was started, with no change in lung function decline post-therapy witnessed in adult patients [40]. These findings mimicked a single centre study which also established that lung function decline may be associated with adherence to therapy longitudinally [41].

There appears to be greater consensus on the sustained reduction in pulmonary exacerbation events longitudinally across studies suggesting an important consideration for the provision of CF services longitudinally. This reduction appears to be most marked in the requirement for intravenous antibiotics. It is currently unclear what the effect of modulator therapy will be on airway microbiology with some studies reporting reductions in pathogen yield. However, it is difficult to control for the marked reduction in spontaneous sputum expectoration in individuals taking these therapies and worldwide registry data will be useful to assess the impact of therapy on acquiring new pathogenic organisms in the lungs.

\section{Multisystem effects}

CF is a multi-organ disease and evidence is emerging on the effects of CFTR modulators beyond the respiratory system. Clinical trial evidence has shown that CFTR modulation will act 
beyond the respiratory system with demonstration of a reduction in sweat chloride as a biomarker of CFTR effect. The clinical implications of this finding may not be profound but would suggest that some individuals can reduce or stop sodium chloride supplementation which is advised for individuals with high sweat losses during warmer weather or in relation to intense exercise, and it may have an influence on the rare complication of Pseudo-Bartter syndrome if administered early in life.

Of greater interest is the influence of highly effective CFTR modulation on nutritional and pancreatic status. Improvements in weight and $\mathrm{BMI}$ are well-recognised in both randomised controlled trials and observational studies of Gly551Asp individuals receiving ivacaftor [11, 42, 43]. Elexacaftor/tezacaftor/ivacaftor triple therapy was shown to increase $\mathrm{BMI}$ and weight in phase 3 trials for both Phe508del heterozygotes and homozygotes [28, 29]. Ivacaftor has also been shown to have a positive impact on linear growth in Gly551Asp individuals aged 6 to 11 years old [43]. These findings are encouraging as nutritional status is a key influence on outcomes in CF. Additionally, pancreatic destruction in CF generally occurs very early in life (usually before 5 years of age) and necessitates the use of supplemental pancreatic enzymes in an effort to decrease problems with malabsorption. Case reports and clinical trials of modulator use in children under 5 years indicate that there may be improvement in pancreatic exocrine function in a proportion of individuals with Gly551Asp or other gating mutations treated with ivacaftor, and Phe508del homozygous individuals treated with lumacaftor/ivacaftor [43]. We await similar reports with elexacaftor/tezacaftor/ivacaftor. The clinical implications of this may be profound with a reduced need for supplemental enteral feeding likely; however, it is also not without caveats. PwCF have been long conditioned to have a high calorie diet and tailored dietary advice may now be needed for individuals who may be placed at risk of the complications of obesity.

A registry-based ivacaftor study reported a reduced prevalence and relative risk of CF-related diabetes in both their US and UK cohorts [44]. However, smaller studies have failed to show a consistent improvement in endogenous insulin production, glucose tolerance, insulin requirements or HbA1c following treatment with ivacaftor [43].

CF-related hepatobiliary disease is a nonspecific, heterogeneous diagnosis encompassing biliary tract disease, biliary cirrhosis, and both cirrhotic and non-cirrhotic liver disease. The registry-based ivacaftor study reported lower prevalence and relative risk of hepatobiliary complications in both their US and UK cohorts [44]. Small studies have indicated that ivacaftor may reduce symptoms of gastro-oesophageal reflux disease, normalise intestinal $\mathrm{pH}$ and reduce intestinal inflammation (indicated by lower faecal calprotectin levels) [43]. However, in a larger study, reduction in prevalence of gastro-intestinal complications failed to reach statistical significance in either the US or UK cohort [44].

CF bone disease is characterised by low bone mineral density. The large registry study reported a reduction in prevalence and relative risk of bone and joint disorders (defined as arthritis/ arthropathy, bone fracture, osteopenia and osteoporosis) in both the US and UK cohorts [44]. A small study reported an improvement in lumbar z-spine scores in Gly551Asp individuals treated with ivacaftor [43].

Given the generalised gains in health reported with CFTR modulators, it seems likely that fertility would improve concurrently. Reports have highlighted normalisation of menstrual cycles as well as both planned and unplanned spontaneous pregnancies in individuals previously unable to conceive naturally $[43,45]$. However, the safety of modulators in pregnancy and lactation remains understudied. Animal models have not shown worrying signals, and a number of case reports and a survey of CF clinicians reporting on a total of 64 pregnancies have been encouraging, with no modulator-related fetal complications reported [45]. Importantly, there have been reports of clinical decline in pregnant mothers following cessation of modulator therapy (based on the unknown risk to the fetus), prompting a resumption of therapy during pregnancy [45].

Figure 3 summarises the pulmonary and multisystem effects of CFTR modulators.

\section{Side-effects and adverse events}

The safety profile of CFTR modulators in trials and beyond has been encouraging [11, 28, 29, 44]. In general, CFTR modulators appear to be well tolerated although the limited timeframe since licensing needs to be acknowledged [46]. In CFTR modulators currently available in clinical practice (ivacaftor, lumacaftor/ivacaftor, tezacaftor/ivacaftor, and elexacaftor/tezacaftor/ivacaftor), the most frequently reported adverse events are pulmonary exacerbations, cough, and increased sputum [47]. With respect to extrapulmonary adverse events, the more frequently reported are headache and diarrhoea [47]. Lumacaftor/ivacaftor had the highest drug discontinuation rate among CFTR modulators approved for clinical use, with $2.8 \%$ of the study cohort discontinuing use [47], but significantly higher rates of discontinuation have been reported in practice [48]. Some areas of concern have included a small but notable risk of significant rises in liver function tests, dictating that blood tests are performed at least quarterly in the first year and annually thereafter. Following on from findings in animal studies, there has been a concern that ivacaftor may give rise to juvenile cataracts, but this has not at this point been deemed a significant problem in humans. 


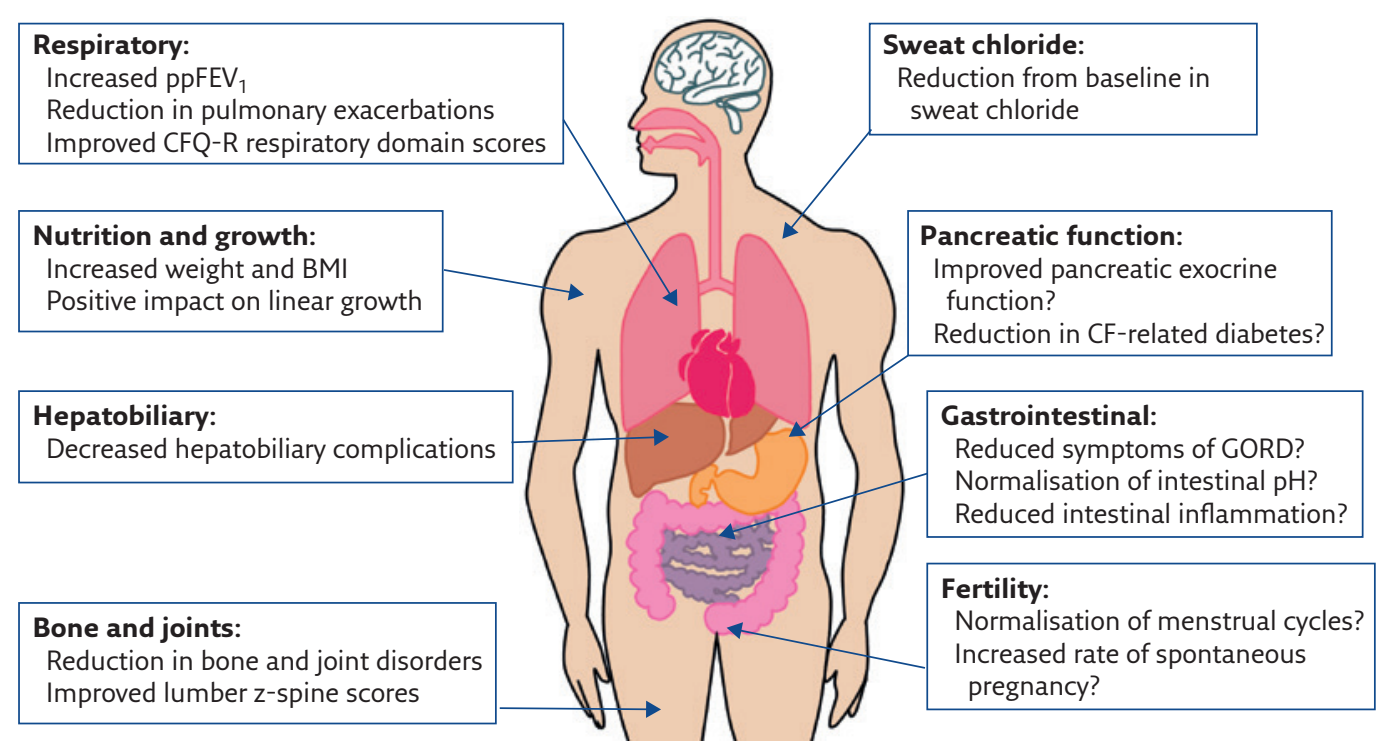

Figure 3 Reported and potential respiratory and multisystem effects of CFTR modulators. GORD: gastro-oesophageal reflux disease.

\section{Drug-drug interactions}

Metabolism of CFTR modulators is primarily by the cytochrome P450 (CYP) family of enzymes. Cytochrome enzymes can be induced or inhibited by a number of drugs, resulting in either enhanced metabolism, faster elimination and lower drug levels; or slower metabolism, slower elimination and increased drug levels, respectively. The P450 subtypes CYP3A4 and CYP3A5 metabolise ivacaftor, tezacaftor and elexacaftor to less active metabolites, leading to lower levels of active drug [49]. Lumacaftor levels are unchanged by CYP3A [49]. Along with a number of other medications (including carbamazepine, phenytoin, rifampicin, gliclazides, barbiturates and glucocorticoids), lumacaftor and, to a lesser extent, ivacaftor induce CYP3A, and so lead to lower levels of active drug. There are a number of drugs that inhibit CYP3A, leading to increased modulator levels, including macrolide antibiotics, azole anti-fungals, amiodarone and grapefruit juice. Lumacaftor also induces and inhibits several other CYP enzymes with the potential for a number of other drug-drug interactions including oral contraceptives, warfarin, fluoxeteine, omeprazole and voriconazole [49]. Elexacaftor has the potential to increase exposure to some statin therapies and caution should be exerted. Importantly no significant impact on hormonal contraception has been witnessed in elexacaftor/ tezacaftor/ivacaftor.

\section{Ongoing challenges}

The introduction of therapies with a profound impact on health status and quality of life of individuals is not without challenges for PwCF and their care teams alike. Although widely welcomed, the psychological impacts of these therapies should not be underestimated, and periods of adjustment may be needed to such a dramatic change in one's health status. PwCF may feel unprepared for life with a potentially changed horizon. School or university absenteeism due to previous ill health may diminish preparedness for some individuals with large clinical benefits. Some may feel regret that this medication was not available sooner before a decline in their own health status or even reflect on peers they may have lost to the condition who did not get the opportunity to see if these treatments worked for them. Some individuals of course will not tolerate these medications, which will pose its own challenges. Psycho-social teams are engrained within CF multidisciplinary care teams and their roles will remain essential as newfound challenges emerge.

Any improvements in survival would further the pre-existing trend of an expanding adult CF population with a greater degree of chronic disease and treatment-related complications. In addition to well recognised extrapulmonary complications such as diabetes and osteoporosis, an increase in survival may lead to the emergence of complications of ageing such as cardiovascular disease or chronic kidney disease due to factors such as diabetes and aminoglycoside use [50]. There is also an increased risk of gastrointestinal malignancies in CF [51], which will become more relevant as the demographics of CF populations change. This will likely alter the nature of work in CF centres as multidisciplinary care teams proactively screen for age-related complications and promote health into middle age and beyond.

The role of current therapies in CF will also need to be actively explored. Whilst it is likely that those 


\section{Self-evaluation questions}

1. The most common class of CF mutations affect CFTR function in which way?

a) No functional CFTR being created (class I)

b) CFTR protein folding and trafficking to the cell membrane (class II)

c) A defective channel gate which diminishes the function of CFTR (class III)

d) The CFTR channel is faulty at the cell membrane (class IV)

e) CFTR is not expressed at the membrane in sufficient quantities (class $\mathrm{V}$ )

f) CFTR has reduced stability at the cell membrane (class VI)

2. The triple CFTR modulator elexacaftor/tezacaftor/ivacaftor is made up of which types of modulators?
a) 2 potentiators and 1 corrector
b) 1 potentiator and 2 correctors
c) 1 potentiator, 1 corrector and 1 stabiliser

3. Which of these extrapulmonary effects of CFTR modulators have not been reported in the literature?
a) Reduction in sweat chloride concentration
b) Increase in body mass index
c) Reduction in anxiety
d) Increase in linear growth

4. Which of the following CFTR modulators would an 18-year-old person with CF heterozygous for Phe508del and W1282X (a MF mutation) be eligible for?
a) Ivacaftor
b) Lumacaftor/ivacaftor
c) Tezacaftor/ivacaftor
d) Elexacaftor/tezacaftor/ivacaftor

5. Which of the following statements is incorrect?
a) CFTR modulators target the underlying defect in cystic fibrosis
b) Clinical trials show a substantial improvement in lung function with CFTR modulators currently licensed for use
c) CFTR modulators have a range of extrapulmonary effects which need careful monitoring
d) All people with CF are currently eligible for CFTR modulators

chronically colonised with airway pathogens will continue to derive some benefit from therapies such as nebulised antibiotics, the role of other therapies such as nebulised mucolytics is less clear and is an active area of study. Great efforts will have to be placed on evaluating current therapies, and the balance between treatment burden and health outcomes for individuals who are benefitting from these therapies.

Another great challenge is the heterogeneous availability of these medications worldwide, with individuals in many countries unable to access therapies due to issues such as licensing and cost. A major priority for the CF community globally is to ensure the "standard of care" can be met for all individuals. Additionally, approximately $10 \%$ of individuals will not benefit from these medications due to their specific gene mutations being unresponsive. Further research is taking place and is acutely needed to find solutions which may offer this population similar advantages to those seen in the majority. Efforts should also be focused on improving the efficacy of these existing therapies as CFTR function is not normalised for the majority of individuals; however, trial design may be challenging and require "washout" periods of existing therapies which will place individuals at risk of decline.

\section{Conclusions}

CFTR modulator therapies currently in clinical practice have had profound impacts on the health and wellbeing of PwCF. Further study is required to fully project the impact that these medications will have longitudinally, but it is likely to become more significant as licensing expands to younger age groups. There remains a key unmet need for a substantial minority of patients who do not qualify for access to these therapies or cannot gain access, which provides key goals for CF care teams into the future.

\section{Key points}

- $C F$ is a multisystem autosomal recessive condition caused by mutations in the CFTR gene, which codes for the CFTR protein.

- Different mutations to the CFTR gene disrupt the normal function of the CFTR channel in different ways.

- Understanding the molecular consequences of specific genetic mutations has resulted in the development and subsequent clinical use of highly effective therapies for a majority of patients with CF.

- CFTR modulator therapies are likely to significantly alter the clinical course and usual management of CF. 


\section{Affiliations}

\section{Daniel H. Tewkesbury1,2, Rebecca C. Robey2,3, Peter J. Barry ${ }^{1,2}$}

1 Manchester Adult Cystic Fibrosis Centre, Wythenshawe Hospital, Manchester University NHS Foundation Trust, Manchester, UK. '2Division of Immunology, Immunity to Infection \& Respiratory Medicine, University of Manchester, Manchester, UK. ${ }^{3}$ The North West Lung Centre, Wythenshawe Hospital, Manchester University NHS Foundation Trust, Manchester, UK.

\section{Conflicts of interest}

P.J. Barry reports receiving consulting fees from Vertex pharmaceuticals, Insmed pharmaceuticals and Gilead, outside the submitted work. Payment or honoraria for lectures, presentations, speakers' bureaus, manuscript writing or educational events received from Vertex pharmaceuticals and Gilead pharmaceuticals, outside the submitted work. D.H. Tewkesbury and R.C. Robey have nothing to disclose.

\section{References}

1. Barben J. First description of cystic fibrosis. J Cyst Fibros 2021: 20: 183.

2. Kerem B, Rommens JM, Buchanan JA, et al. Identification of the cystic fibrosis gene: genetic analysis. Science 1989; 245: 1073-1080.

3. Riordan JR, Rommens JM, Kerem B, et al. Identification of the cystic fibrosis gene: cloning and characterization of complementary DNA. Science 1989; 254: 1066-1073.

4. Rommens JM, Iannuzzi MC, Kerem B, et al. Identification of the cystic fibrosis gene: chromosome walking and jumping. Science 1989; 245: 1059-1065

5. CFTR2. CFTR2 Variant List History. https://cftr2.org/ mutations_history Date last accessed: 16 June 2021. Date last updated: 31 July 2020.

6. Welsh MJ, Smith AE. Molecular mechanisms of CFTR chloride channel dysfunction in cystic fibrosis. Cell 1993; 73: 1251-1254.

7. Rowe SM, Miller S, Sorscher EJ. Cystic fibrosis. N Engl J Med 2005; 352: 1992-2001.

8. Veit G, Avramescu RG, Chiang AN, et al. From CFTR biology toward combinatorial pharmacotherapy: expanded classification of cystic fibrosis mutations. Mol Biol Cell 2016; 27: 424-433.

9. Van Goor F, Hadida S, Grootenhuis PDJ, et al. Rescue of CF airway epithelial cell function in vitro by a CFTR potentiation, VX-770. Proc Natl Acad Sci USA 2009; 106: 18825-18830.

10. Accurso FJ, Rowe SM, Clancy JP, et al. Effect of VX-770 in persons with cystic fibrosis and the G551D-CFTR mutation. N EnglJ Med 2010; 363: 1991-2003.

11. Ramsey BW, Davies J, McElvaney NG, et al. A CFTR Potentiator in patients with cystic fibrosis and the G551D mutation. N EnglJ Med 2011; 365: 1663-1672.

12. Flume PA, Liou TG, Borowitz DS, et al. Ivacaftor in subjects with cystic fibrosis who are homozygous for the F508del-CFTR mutation. Chest 2012; 142: 718-724.

13. De Boeck K, Munck A, Walker S, et al. Efficacy and safety of ivacaftor in patients with cystic fibrosis and a non-G551D gating mutation. J Cyst Fibros 2014; 13: 674-680.

14. Moss RB, Flume PA, Elborn JS, et al. Efficacy and safety of ivacaftor in patients with cystic fibrosis who have an Arg117His-CFTR mutation: a double-blind, randomised controlled trial. Lancet Respir Med 2015; 3: 524-533.

15. Durmowicz AG, Lim R, Rogers H, et al. The U.S. Food and drug administration's experience with ivacaftor in cystic fibrosis. establishing efficacy using in vitro data in lieu of a clinical trial. Ann Am Thorac Soc 2018; 15: 1-2.

16. Harbeson SL, Morgan AJ, Liu JF, et al. Altering metabolic profiles of drugs by precision deuteration 2: discovery of a deuterated analog of ivacaftor with differentiated pharmacokinetics for clinical development. J Pharmacol Exp Ther 2017; 362: 359-367.

17. Yeh $\mathrm{HI}$, Sohma $\mathrm{Y}$, Conrath $\mathrm{K}$, et al. A common mechanism for CFTR potentiators. J Gen Physiol 2017; 149: 1105-1118.
18. Kazani S, Rowlands DJ, Bottoli I, et al. Safety and efficacy of the cystic fibrosis transmembrane conductance regulator potentiator icenticaftor (QBW251). J Cyst Fibros 2021; 20: 250-256.

19. Rowe SM, Jones I, Dransfield MT, et al. Efficacy and safety of the CFTR potentiator icenticaftor (QBW251) in COPD: results from a phase 2 randomized trial. Int J Chron Obstruct Pulmon Dis 2020; 15: 2399-2409.

20. Van Goor F, Straley KS, Cao D, et al. Rescue of DeltaF508-CFTR trafficking and gating in human cystic fibrosis airway primary cultures by small molecules. Am J Physiol Lung Cell Mol Physiol 2006; 290: L1117-L1130.

21. Van Goor F, Hadida S, Grootenhuis PDJ, et al. Correction of the F508del-CFTR protein processing defect in vitro by the investigational drug VX-809. Proc Natl Acad Sci USA 2011; 108: 18843-18848.

22. Wainwright CE, Elborn JS, Ramsey BW, et al. Lumacaftorivacaftor in patients with cystic fibrosis homozygous for Phe508del CFTR. N Engl J Med 2015; 373: 220-231.

23. Taylor-Cousar JL, Munck A, McKone EF, et al. Tezacaftorivacaftor in patients with cystic fibrosis homozygous for Phe508del. N EnglJ Med 2017; 377: 2013-2023.

24. Munck A, Kerem E, Ellemunter $\mathrm{H}$, et al. Tezacaftor/ivacaftor in people with cystic fibrosis heterozygous for minimal function CFTR mutations. J Cyst Fibros 2020; 19: 962-968.

25. McKone EF, DiMango EA, Sutharsan S, et al. A phase 3 , randomized, double-blind, parallel-group study to evaluate tezacaftor/ivacaftor in people with cystic fibrosis heterozygous for F508del-CFTR and a gating mutation. J Cyst Fibros 2021; 20: 234-242.

26. Rowe SM, Daines C, Ringshausen FC, et al. Tezacaftor-ivacaftor in residual-function heterozygotes with cystic fibrosis. $N$ Engl J Med 2017; 377: 2024-2035.

27. Schwarz C, Sutharsan S, Epaud R, et al. Tezacaftor/ivacaftor in people with cystic fibrosis who stopped lumacaftor/ivacaftor due to respiratory adverse events. J Cyst Fibros 2021; 20: 228-233.

28. Heijerman HGM, McKone EF, Downey DG, et al. Efficacy and safety of the elexacaftor plus tezacaftor plus ivacaftor combination regimen in people with cystic fibrosis homozygous for the F508del mutation: a double-blind, randomised, phase 3 trial. Lancet 2019; 394: 1940-1948.

29. Middleton PG, Mall MA, Dřevínek P, et al. Elexacaftortezacaftor-ivacaftor for cystic fibrosis with a single Phe508del allele. N Engl J Med 2019; 381: 1809-1819.

30. Griese M, Costa S, Linnemann RW, et al. Safety and efficacy of elexacaftor/tezacaftor/ivacaftor for 24 weeks or longer in people with cystic fibrosis and one of more F508del alleles: interim results of an open-label phase 3 clinical trial. $\mathrm{Am} J$ Respir Crit Care Med 2021; 203: 381-385.

31. Barry PJ, Mall MA, Alvarez A, et al. Triple therapy for cystic fibrosis Phe508del-gating and -residual function genotypes. N EnglJ Med 2021; 385: 815-825.

\section{Suggested answers}

1. b.

2. b.

3. c.

4. d.

5. d. 
32. Bell SC, Barry PJ, De Boeck K, et al. CFTR activity is enhanced by the novel corrector GLPG2222, given with and without ivacaftor in two randomized trials. J Cyst Fibros 2019; 18 700-707

33. Matos AM, Gomes-Duarte A, Faria M, et al. Prolonged co-treatment with HGF sustains epithelial integrity and improves pharmacological rescue of Phe508del-CFTR. Sci Rep 2018; 8: 13026.

34. Lobo MJ, Amaral MD, Zaccolo M, et al. EPAC1 activation by CAMP stabilizes CFTR at the membrane by promoting its interaction with NHERF1. J Cell Sci 2016; 129: 2599-2612.

35. Zaman K, Sawczak V, Zaidi A, et al. Augmentation of CFTR maturation by S-nitrosoglutathione reductase. Am J Physiol Lung Cell Mol Physiol 2016; 310: L263-L270.

36. Giuliano KA, Wachi S, Drew L, et al. Use of a high-throughput phenotypic screening strategy to identify amplifiers, a nove pharmacological class of small molecules that exhibit functional synergy with potentiators and correctors. SLAS Discov 2018; 2: 111-121.

37. Burgel PR, Durieu I, Chiron R, et al. Rapid improvement after starting elexacaftor-tezacaftor-ivacaftor in patients with cystic fibrosis and advanced pulmonary disease. Am J Respir Crit Care Med 2021; 204: 64-73.

38. Sawicki GS, Dasenbrook E, Fink AK, et al. Rate of uptake of ivacaftor use after U.S. Food and drug administration approva among patients enrolled in the U.S. Cystic fibrosis foundation patient registry. Ann Am Thorac Soc 2015; 12: 1146-1152.

39. Volkova N, MoyK, Evans J, et al. Disease progression in patients with cystic fibrosis treated with ivacaftor: data from national US and UK registries. J Cyst Fibros 2020; 19: 68-79.

40. Kirwan L, Fletcher $G$, Harrington M, et al. Longitudinal trends in real-world outcomes after initiation of ivacaftor. a cohort study from the cystic fibrosis registry of ireland. Ann Am Thorac Soc 2019; 16: 209-216.

41. Mitchell RM, Jones AM, Stocking K, et al. Longitudinal effects of ivacaftor and medicine possession ratio in people with the Gly551Asp mutation: a 5-year study. Thorax 2021; 76 874-879.

42. Habib AR, Kajbafzadeh M, Desai S, et al. A systematic review of the clinical efficacy and safety of CFTR modulators in cystic fibrosis. Sci Rep 2019; 9: 7234.

43. Sergeev V, Chou FY, Lam GY, et al. The extrapulmonary effects of cystic fibrosis transmembrane conductance regulator modulators in cystic fibrosis. Ann Am Thorac Soc 2020; 17 : 147-154.

44. Bessonova L, Volkova N, Higgins M, et al. Data from the US and UK cystic fibrosis registries support disease modification by CFTR modulation with ivacaftor. Thorax 2018; 73: 731-740.

45. Taylor-Cousar JL. CFTR Modulators: impact on fertility, pregnancy, and lactation in women with cystic fibrosis. J Clin Med 2020; 9: 2706.

46. Dagenais RVE, Su VCH, Quon BS. Real-world safety of CFTR modulators in the treatment of cystic fibrosis: a systematic review. J Clin Med 2020; 10: 23.

47. Gramegna A, Contarini M, Aliberti S, et al. From ivacaftor to triple combination: a systematic review of efficacy and safety of CFTR modulators in people with cystic fibrosis. Int J Mol Sci 2020; 21: 5882

48. Jennings MT, Dezube R, Paranjape S, et al. An observational study of outcomes and tolerances in patients with cystic fibrosis initiated on lumacaftor/ivacaftor. Ann Am Thorac Soc 2017; 14: 1662-1666

49. Shteinberg M, Taylor-Cousar JL. Impact of CFTR modulator use on outcomes in people with severe cystic fibrosis lung disease. Eur Respir Rev 2020; 29: 190112.

50. Berg KH, Ryom L, Faurholt-Jepsen D, et al. Prevalence and characteristics of chronic kidney disease among Danish adults with cystic fibrosis. J Cyst Fibros 2018; 17: 478-483.

51. Yamada A, Komaki Y, Komaki F, et al. Risk of gastrointestinal cancers in patients with cystic fibrosis: a systematic review and meta-analysis. Lancet Oncol 2018; 19: 758-767.

52. Fuchs HJ, Borowitz DS, Christiansen DH, et al. Effect of aerosolized recombinant human DNase on exacerbations of respiratory symptoms and on pulmonary function in patients with cystic fibrosis. N Engl J Med 1994; 331: 637-642.

53. Ramsey BW, Pepe MS, Quan JM, et al. Intermittent administration of inhaled tobramycin in patients with cystic fibrosis. N EnglJ Med 1999; 340: 23-30.

54. Saiman L, Marshall BC, Mayer-Hamblett N, et al. Azithromycin in patients with cystic fibrosis chronically infected with Pseudomonas aeruginosa: a randomized controlled trial. JAMA 2003; 290: 1749-1756.

55. Elkins MR, Robinson M, Rose BR, et al. A controlled trial of long-term inhaled hypertonic saline in patients with cystic fibrosis. N Engl J Med 2006; 354: 229-240.

56. McCoy KS, Quittner AL, Oermann CM, et al. Inhaled aztreonam lysine for chronic airway Pseudomonas aeruginosa in cystic fibrosis. Am J Respir Crit Care Med 2008; 178: 921-928.

57. Elborn JS, Geller DE, Conrad D, et al. A phase 3, open-label, randomized trial to evaluate the safety and efficacy of levofloxacin inhalation solution (APT-1026) versus tobramycin inhalation solution in stable cystic fibrosis patients. J Cyst Fibros 2015; 14: 507-514 